\title{
Laser interference fabrication of large-area functional periodic structure surface
}

(C) The Author(s) 2018. This article is published with open access at link.springer.com and journal.hep.com.cn

\begin{abstract}
Functional periodic structures have attracted significant interest due to their natural capabilities in regulating surface energy, surface effective refractive index, and diffraction. Several technologies are used for the fabrication of these functional structures. The laser interference technique in particular has received attention because of its simplicity, low cost, and high-efficiency fabrication of large-area, micro/nanometer-scale, and periodically patterned structures in air conditions. Here, we reviewed the work on laser interference fabrication of large-area functional periodic structures for antireflection, self-cleaning, and superhydrophobicity based on our past and current research. For the common cases, four-beam interference and multi-exposure of two-beam interference were emphasized for their setup, structure diversity, and various applications for antireflection, self-cleaning, and superhydrophobicity. The relations between multi-beam interference and multi-exposure of two-beam interference were compared theoretically and experimentally. Nanostructures as a template for growing nanocrystals were also shown to present future possible applications in surface chemical control. Perspectives on future directions and applications for laser interference were presented.
\end{abstract}

Keywords laser interference, four-beam interference, multi-exposure of two-beam interference, additive fabrication

\section{Introduction}

Periodic structures have recently attracted significant interest due to their natural capabilities in regulating

Received October 23, 2017; accepted November 17, 2017

Lei WANG, Zi-Han WANG, Yan-Hao YU, Hong-Bo SUN ( $ه$ )

State Key Laboratory on Integrated Optoelectronics, College of Electronic Science and Engineering, Jilin University, Changchun 130012, China

E-mail: hbsun@jlu.edu.cn surface energy, surface effective refractive index, diffraction, superhydrophobicity, antireflection, and structural colors for various applications [1-5]. Various technologies, such as photolithography [6,7], self-assembly [8], electrospinning [9], and nanoimprinting [6], have been developed for the fabrication of these functional micro/nanostructure arrays. However, the requirements for highly trained operators, expensive cleanrooms, and high-resolution masks have affected the widespread applications of such technologies in the research area, thereby necessitating the development of a simple, low-cost, and high-efficiency method. Laser interference [5,10-13] is a powerful technique for the fabrication of large-area, micro/nanometer-scale, and periodically patterned structures. Laser interference demonstrates micro/nanostructures in air conditions by using a self-redistributed light intensity directly recorded in light-sensitive materials rather than using expensive masks [12]. Flexible structures, such as 1D gratings [14], 2D pillars/holes [15], and 3D crystal-like structures [16], have been produced.

The setup of laser interference is relatively simple, with 2/3/4-beam interferences being the common cases $[5,17-$ 19]. Split beams from a coherent laser interfering on the sample are used to form a redistributed fringe-like intensity. The intensity period for the recorded structures is determined by laser wavelength $\lambda$, refractive index $n$, and the angle between the two beams, $\theta$. The laser wavelength is usually determined by photoresist linear absorption. Most laser sources are reported at 355 [15], $325[11,20], 266$ [5], 257 [21], and $193 \mathrm{~nm}$ at deep ultraviolet wavelengths [22], which limit the resolution of the structures with a minimum period of $\lambda / 2 n$. Thus far, the minimum period of $90 \mathrm{~nm}$ has been realized by the immersion method with a $45 \mathrm{~nm}$ half-pitch and two views of self-aligned frequency-doubled patterns at $22 \mathrm{~nm}$ halfpitch, followed by two potassium hydroxide $(\mathrm{KOH})$ etch pattern-transfer steps [22]. Ultra-short pulsed lasers, such as nanosecond and femtosecond lasers [23], have also been used for laser interference. Considering the coherent length limitation, nanosecond laser sources are typically used in 
the fabrication of high-depth/width-ratio structures. The flexible tuning of beam angle widens the period from microscale to nanoscale and promotes its widespread applications in surface science [5]. With the detailed multibeam interference and multi-exposure of two-beam interference, structures such as microlenses [10], photonic quasi-lattices [24], and micro/nanopillars (micro/nanoholes) $[18,25]$, and several micro/nanostructures based on these templates $[11,16]$ are easily demonstrated.

Research on laser interference is relatively pioneering and promising. Our research group has been extensively involved in this research area. In this study, we present our past and current work on laser interference for the fabrication of large-area functional periodic structure surfaces and provide our perspective on the future of this area. This study includes structural and functional diversity by four-beam interference, multi-exposure of two-beam interference, and the relationship between the multiexposure of two-beam and multi-beam interferences.

\section{Fabrication of functional structures by four-beam interference}

Four-beam interference is one of the interference techniques used for the fabrication of large-area functional structures due to its outstanding capability in one-step realization of $2 \mathrm{D} / 3 \mathrm{D}$ structures $[15,16,18,25-28]$. As shown in Fig. 1(a) [29], four beams numbered $K_{1}$ to $K_{4}$ were found on the sample in which they would split and reflect to interfere with a periodic light intensity of Fig. 1(b). Through a photo-crosslinking process, the corresponding structures form under light distribution with period $\Lambda=\sqrt{2} \lambda / 2 \sin \theta$. Usually, the angles between each
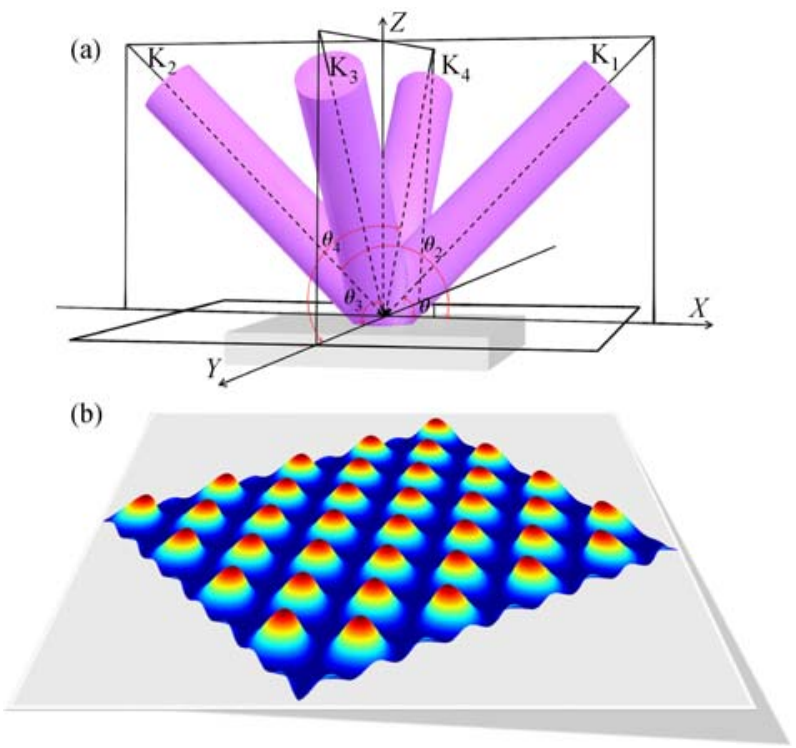

Fig. 1 (a) Set-up of four-beam interference, and (b) its light intensity distribution [29] beam and substrate, which were labeled as $\theta_{1}$ to $\theta_{4}$, were set to limit the structure period, and the angles between the beams determined their distributions in different directions. To adjust the angles, various setups that combined beam splitters and reflectors were used to obtain four beams as $\mathrm{K}_{1}$ to $\mathrm{K}_{4}$. Although the periods in theory were at least $\sqrt{2} \lambda / 2$, the maximum angles of reflectors set for $K_{1}$ to $\mathrm{K}_{4}$ were usually around $70^{\circ}$, which limited the period to the lowest point around $0.8 \lambda$. Meanwhile, the angles of minimum reflectors were usually $1^{\circ}-2^{\circ}$, which resulted in a maximum period of $20 \lambda-30 \lambda$. To fulfill the equal retardance for each beam, laser beam was split at least three times. Although the light path becomes much more complex, it provided more flexible structures by finer control over the power and exposure time of each beam. In comparison, a pyramid prism with four sides and fixed angles is a simple method to realize four-beam interference by applying one incident light beam vertically to the flat bottom [30]. The laser fluences and exposure time for each beam determined the structure surface morphologies. Sample rotation is another way to increase the complexity but is not common in four-beam interference.

\subsection{Structure diversity by four-beam interference}

Four-beam interference has become a powerful tool in the fabrication of various $2 \mathrm{D}$ structures by changing the angle and exposure fluence for each beam $[15,18,26]$. For example, when the intensity of beams $\mathrm{K}_{2}$ and $\mathrm{K}_{4}$ is lower than that of $K_{1}$ and $K_{3}$, the structures become stretched in the parallel direction under anisotropic light distributions shown in (i) and (iii) of Fig. 2(a) [31,32]. In extreme cases, four-beam interference becomes two-beam interference in (ii) and (iv) of Fig. 2(a) by blocking the beams of $\mathrm{K}_{2}$ and $\mathrm{K}_{4}$.

As shown in (i) of Fig. 2(b), elliptical structures with a height less than $1 \mu \mathrm{m}$, shown in (iii) of Fig. 2(b), were obtained by the artifice in Fig. 2(a). The structures bent toward each other with the increase in height. If the height was between 1 and $1.9 \mu \mathrm{m}$, the pillars bent along the shortaxis direction and formed regular "S" shape arrays. If the height increased to $2.6 \mu \mathrm{m}$, then the opposite pillars attracted each other to form a chain-like array, as shown in (iv) of Fig. 2(b). The shape could also be changed by changing the laser fluence. The increased space between the pillars with the laser fluence resulted in a titled angle from $80^{\circ}$ to $45^{\circ}$, which is a similar change to the height. Scanning electron microscope (SEM; JEOL 6700F) images of the pillar arrays for 1.8 and $1.4 \mathrm{~mJ} / \mathrm{cm}^{2}$ are shown in (ii) and (iii) of Fig. 2(c). The important factor for laser fluence is the width of formed structures, which was directly observed from discrete to chain-like in (iv) of Fig. 2(b). The distributions of pillars also affected the structure morphology and its tilt angle. The shapes could also be changed by tuning the space and angles between the structures and laser fluence (Fig. 2(c)).

These structures were used due to the supporting force 


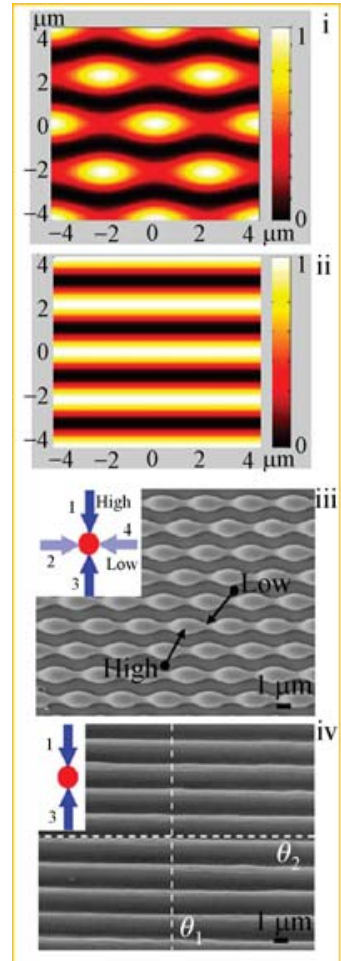

(a)

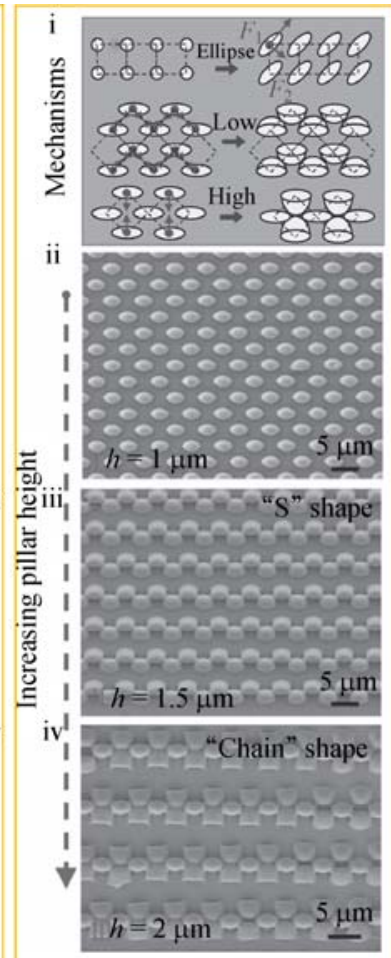

(b)
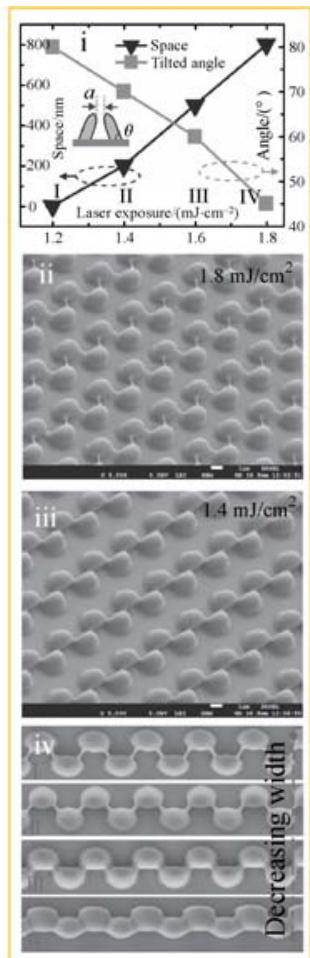

(c)
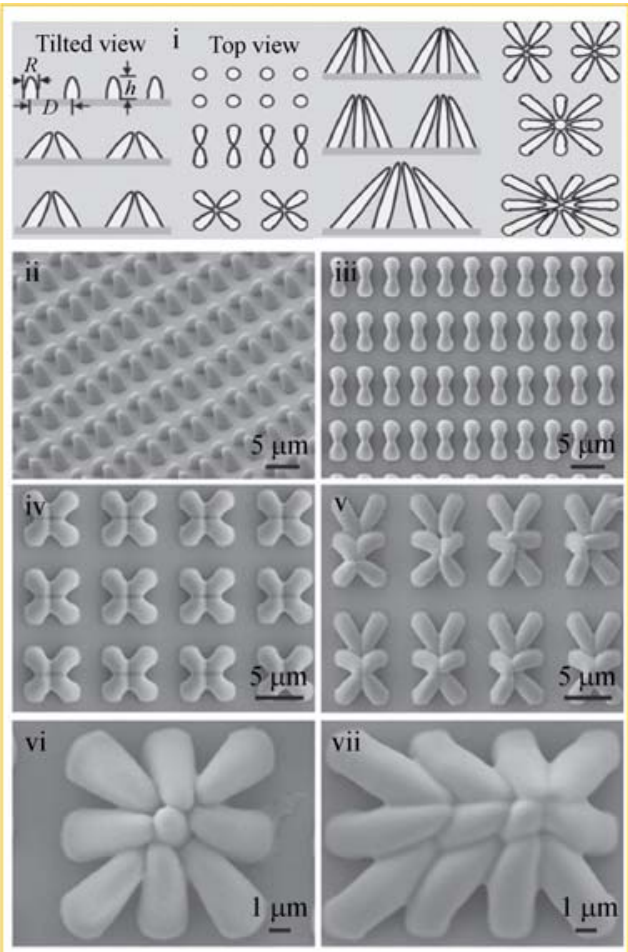

(d)

Fig. 2 Structure diversity by improved four-beam interference [31,32]. (a) Micropearl arrays; (b) anisotropic regular "S" and "chainlike" microstructures prepared by controlling the width of the pillars; (c) a series of complex elliptical pillars realized by controlling the width; (d) regular controllable microstructures realized by capillary force assembly of pillar arrays with different heights

of the materials. A supporting force $F_{\mathrm{s}}-E R^{4} d / h^{3}$ exists, which is defined as the critical force that causes pillar bending [32]. The ratio, $K=F_{\text {att }} / F_{\mathrm{s}}-(2 \pi \gamma / E d) \cos (\alpha / \delta) h^{4} / R^{3}$ [32], for the bending properties of pillar arrays is significantly affected by height $(h)$ and width $(R)$. The pillar stood straight and did not bend at $K<1$. When $K$ became large, the pillars began to bend, and 2-pillar-cell arrays were obtained, as shown in (ii) and (iii) of Fig. 2(d). Experimentally, 4-pillar cell arrays were assembled at $K=$ 3.16, as shown in (iv) of Fig. 2(d). Six-cell, nine-cell, and twelve-cell arrays were simultaneously achieved by increasing the height to 5.3, 6.9, and $8.6 \mu \mathrm{m}$, respectively.

\subsection{Superhydrophobic structures by four-beam interference}

The general pinned and roll-down states in superhydrophobicity are usually determined by structure interval. Stretchable or bent structures are preferred for state transition. As shown in Fig. 3(a), in situ switching from the pinned to the roll-down state was evident due to the curvature of the surface viewed in macro $[18,19]$. Structure arrays were fabricated by four-beam interference with a contact angle of $150^{\circ} \pm 2^{\circ}$. The contact angle slightly increased to $156^{\circ}$ with a radius of curvature of $3.38 \mathrm{~mm}$ or $0.3 \mathrm{~mm}^{-1}$. The relationship between the radius and contact angle is shown in (vi) of Fig. 3(a). Interestingly, the rolling angles for the flat and bent surfaces were different, which resulted in a transition from the pinned state to the rolldown state, as shown in (i) and (ii) of Fig. 3(a). A detailed comparison between flat and curved pillar arrays is presented in Fig. 3(b). The water droplet on a flat pillar array did not roll down at the pinned state when the surface was tilted at $90^{\circ}$ or $180^{\circ}$. However, the water droplet rolled down quickly on a tilted curved surface (curvature ca. 0.5 $\mathrm{mm}^{-1}$ ) and did not stay on a surface with large curvature (curvature ca. $0.7 \mathrm{~mm}^{-1}$ ), especially when the surface was nearly horizontal.

Gecko-foot-like structures were fabricated by four-beam interference with the assistance of photolithography to introduce structures in the order of tens of microns, as shown in Fig. 3(c) [19]. The larger period and smaller period structures were superhydrophilic and superoleophilic in the air environment. However, the structures became superoleophobic in water with contact angles of $150^{\circ}$ and $164^{\circ}$. The contact angle was increased to $175^{\circ}$ by combining the two types of structures. At this angle, the oil could not remain, especially when the sample surface was nearly horizontal. With this sample, the separation of oil from water was easily demonstrated by immersing the mixture into water to sweep the oil. 

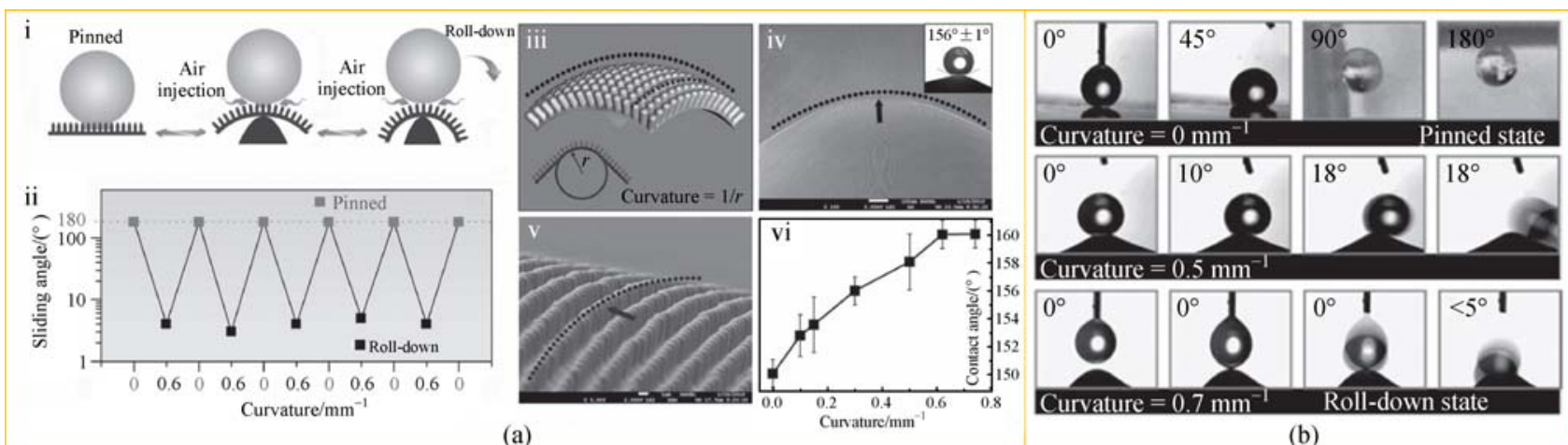

(a)

(b)
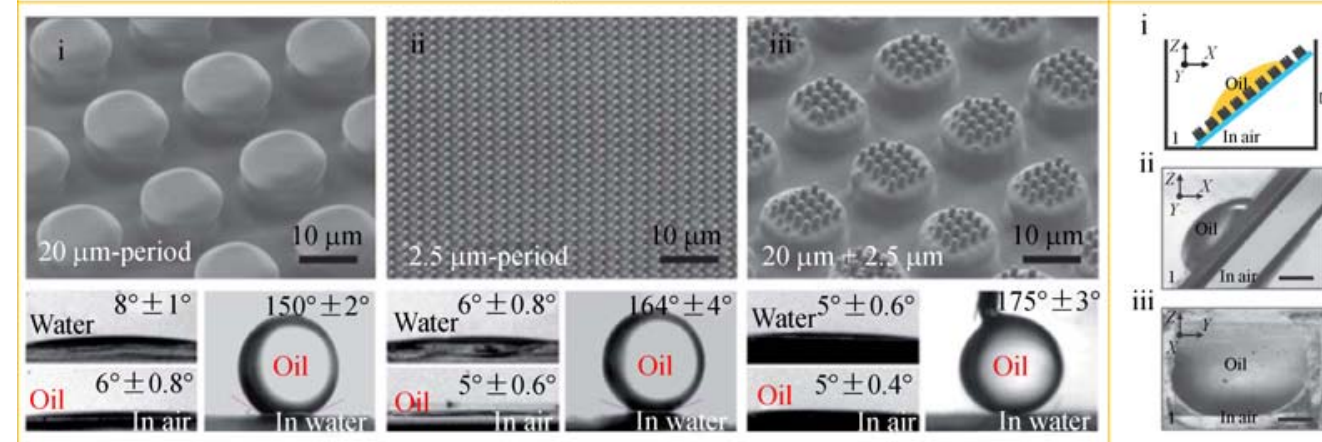

(c)

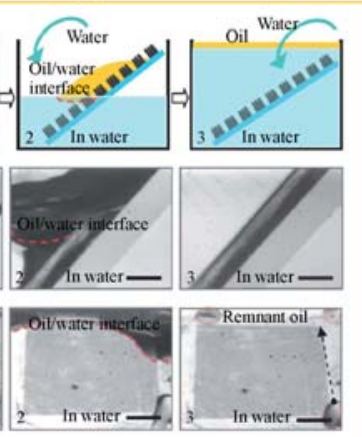

(d)

Fig. 3 (a) Reversible switching of three superhydrophobic states by curvature change and reversible tests for the switching between the pinned and roll-down states; (b) the corresponding state changing in experiment [18]; (c) tilted scanning electron microscopy images of $20 \mu \mathrm{m}$ period, $2.5 \mu \mathrm{m}$ period pillar arrays, and hierarchical gecko foot-like microstructures; (d) the contact angle in air and water, and their self-cleaning anti-oil capability on extreme superoleophobic surfaces [19]

\section{Fabrication of functional structures by multi-exposure of two-beam interference}

Four-beam interference is a powerful tool in the fabrication of various structures. Interference by many beams has become significant due to the interesting patterns and various applications on 3D photonic structures $[33,34]$. However, the setup becomes complicated with the increase in the number of beams, which resulted in many alignment errors. To avoid these issues, multi-exposure of two-beam interference was proposed due to its flexibility and simple setup, as shown in Fig. 4 [5,12,13,35-37]. In Fig. 4, the relationship between multiple exposure two-beam interference and multi-beam interference is first introduced [12]. Biomimetic hierarchical 3D textures were obtained by angle-multiplexed optical printing, which have a comparable aspect ratio, with a period spanning from 4 $\mu \mathrm{m}$ to $300 \mathrm{~nm}$ (more than one order of magnitude), and the height spanning from $0.9 \mu \mathrm{m}$ to $40 \mathrm{~nm}$ [5]. The structure surface area was expanded by moving the motor during the fabrication process, which provided a rapid method for large-area surface texturing [5,38].

3.1 Relationship between multi-beam interference and multi-exposure of two-beam interference

On the basis of the interference theory, the light intensity for each beam is shown in Eq. (1) [12,37],

$$
\begin{aligned}
\vec{U}_{j}(\vec{P}, t) & =\vec{A}_{j}(\vec{P}) \mathrm{e}^{-i\left[\omega_{j} t-\varphi_{j}(\vec{P})\right]} \\
& =\vec{A}_{j}(\vec{P}) \mathrm{e}^{i \varphi_{j}(\vec{P})} \mathrm{e}^{-i \omega_{j} t}, j=1,2,3 \ldots,
\end{aligned}
$$

where $\vec{U}$ represents the vector wave, $\vec{A}$ is the amplitude, $\omega$ is the frequency, $\varphi$ is the initial phase, and $j$ represents the order of the split beam. The redistributed light intensity for $N$ th beam is shown in Eqs. (2) and (3) [12],

$$
\begin{aligned}
I_{N}(P)= & \sum_{j=1}^{N} I_{j}(\vec{P}) \\
& +\sum_{j=1}^{N} \sum_{k=1, j \neq k}^{N} \sqrt{I_{j}(\vec{P}) I_{k}(\vec{P}) \cos \left[\delta_{j-k}(\vec{P})\right]},
\end{aligned}
$$

where

$$
\left\{\begin{array}{l}
I_{j}(\vec{P})=\vec{U}_{j}(\vec{P}) \vec{U}_{j}^{*}(\vec{P})=A_{j}^{2}(\vec{P}) \\
\delta_{j-k}(\vec{P})=\varphi_{j}(\vec{P})-\varphi_{k}(\vec{P})
\end{array}\right.
$$




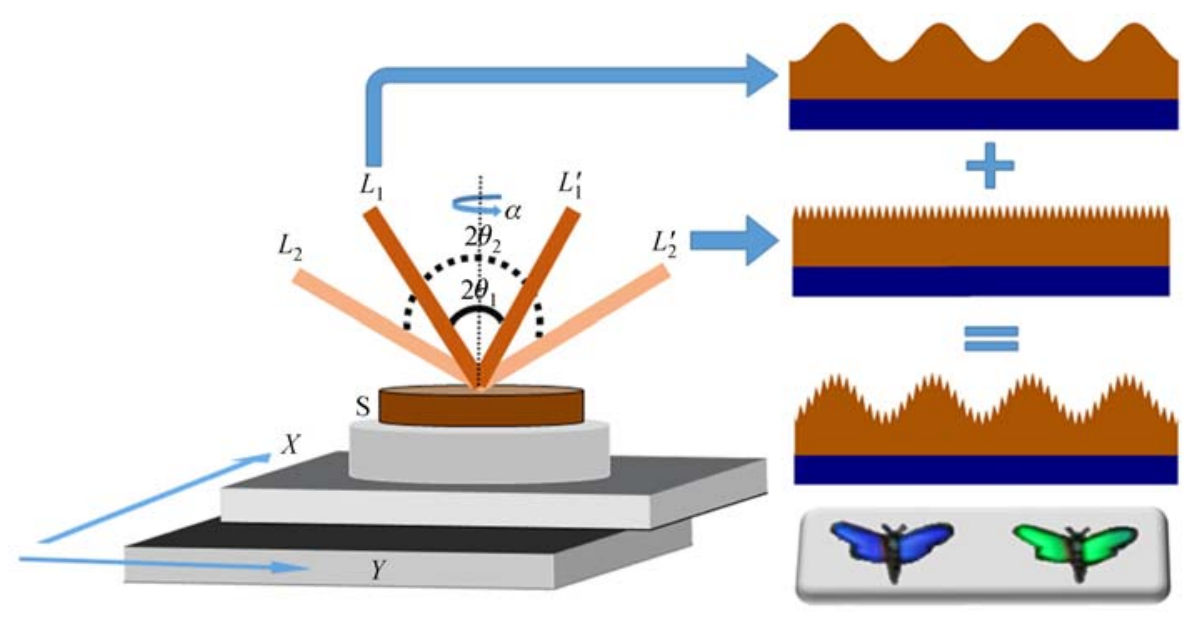

Fig. 4 Schematic for fabrication of hierarchical 3D textures by angle varied multiple exposure of 2-beam interference process. $L_{1} L_{1}^{\prime}$ is the exposure for the larger period, and $L_{2} L_{2}^{\prime}$ is the exposure for the smaller period [5]

If $n$ exposures of $N$-beam interference are found, then the light intensity is expressed as Eq. (4) [12],

$$
I_{N}^{n}=\sum_{j=1}^{n} I_{N}\left(P_{j}\right),
$$

where $n$ exposures are usually attained by rotating the sample by $\theta_{j} 180^{\circ} / n$, as shown in Eqs. (5) and (6) [12],

$$
\begin{gathered}
\theta_{j}=\frac{180^{\circ}(j-1)(n-1)}{n}, \\
P_{j}=P_{1}\left[\cos \theta_{j}, \sin \theta_{j}\right] .
\end{gathered}
$$

For example, the light intensity of four-beam interference by setting $N=4$ and $n=1$ is shown in Fig. 1(b). Similarly, $N=2$ represents the two-beam interference. The intensity of multi-beam interference and multi-exposure of two-beam interference are shown in Fig. 5 [12]. A systematic comparison of the images shown in Fig. 5 shows the following relationship between multi-beam interference and multi-exposure of two-beam interference [12]: 1) Patterns made by multi-beam interference and multi-exposure of two-beam interference are of $n$-fold symmetry; (2) the intensity distribution is the same for two interferences, but the maximum intensity of multiexposure of two-beam interference is much less than that of multi-beam interference; 3 ) a singular point appears in the center of multi-beam interference pattern but none in multi-exposure of two-beam interference. Energies are collected at the center if many beams are used in MBI, and a relatively well-proportioned intensity distribution in a large area is formed by METBI; 4) a $2 n$-exposure MBI can be replaced by an $n$-exposure METBI if large-area wellproportioned structures of $n$-fold symmetry are required.

The multi-exposure of two-beam interference flow is shown as follows: Potential 1D gratings were formed by the first exposure of two-beam interference. The second exposure was conducted by rotating the sample by $\theta_{j}$, as defined in Eq. (5). Then, the third exposure and other exposures were processed by the same method. In the completion of all exposures, structures of $n$-fold symmetry were obtained after developing, as shown in (iii) of Figs. 5(a)-5(f). Furthermore, several techniques for multiexposure of two-beam interference and multi-beam interference were found by combining the laser fluence for each beam and exposure time [37]. However, exchanging the two technologies was possible, thereby decreasing the difficulty of the fabrication process.

3.2 Biomimetic hierarchical 3D textures by angle-multiplexed multi-exposure of two-beam interference

Natural superhydrophobic and antireflective structures constantly have multi-scales, including micro-features and nanofeatures [39]. However, manufactured multi-scaled structures using common technology are difficult to achieve. Complex steps with masks are the usual means by which these structures are achieved. These methods require high cost and have low efficiency. In this subsection, the production of biomimetic hierarchical 3D textures was demonstrated by angle-multiplexed multi-exposure of two-beam interference. Only the multi-exposure of two-beam interference was conducted for structures with the same level. However, the angles of exposure were tuned as the calculated period for structures with different levels, followed by the same flow of multi-exposure of two-beam interference. In this method, biomimetic hierarchical 3D textures that have comparable aspect ratios with a period spanning from $4 \mu \mathrm{m}$ to $300 \mathrm{~nm}$ (more than one order of magnitude) and height spanning from $0.9 \mu \mathrm{m}$ to $40 \mathrm{~nm}$ were obtained, as shown in Fig. 6(a). The multi-scale biomimetic hierarchical 3D structures possessed superhydrophobicity, angle-dependent diffraction, and structural color capacities, as shown in Figs. 6(b) and $6(\mathrm{c})[5]$. 


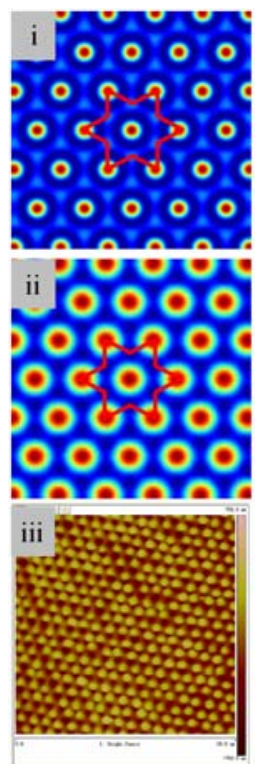

(a)
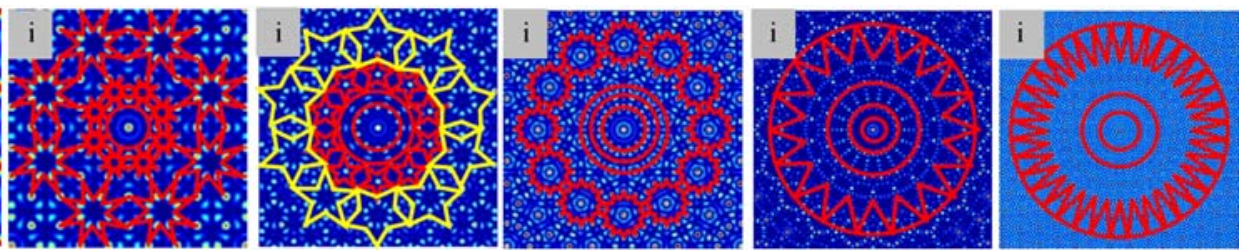

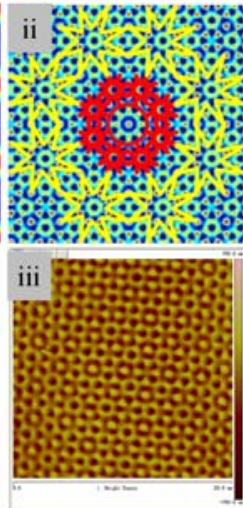

(b)

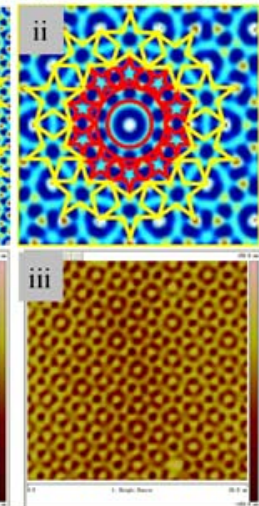

(c)
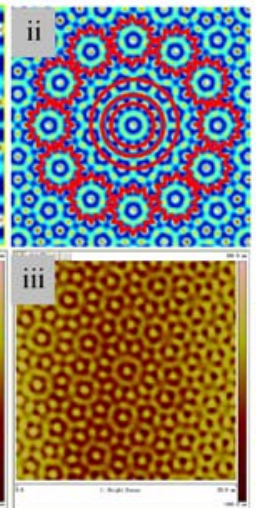

(d)

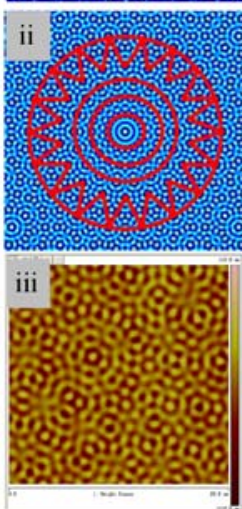

(e)
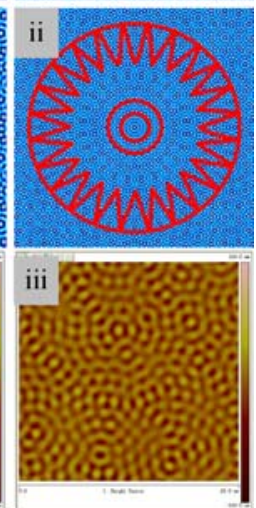

(f)

Fig. 5 Shown in the upper row (i) of (a)-(f) are theoretical patterns made by 6-, 8-, 10-, 12-, 24-, and 36-beam interference; (ii) and (iii) of (a)-(f) are theoretical and experimental atomic force microscope images made by 3, 4, 5, 6, 12, 18 exposures of two-beam interference [12]
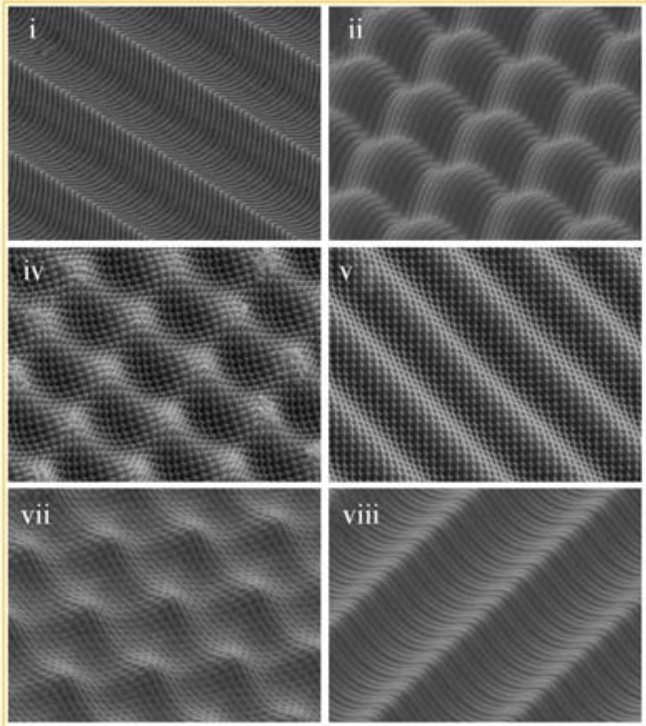

(a)
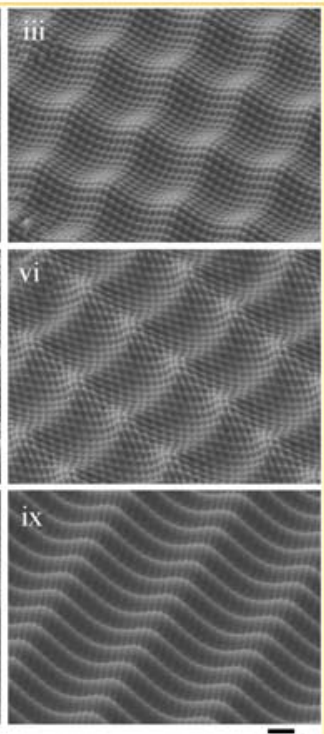

$\overline{1 \mu \mathrm{m}}$

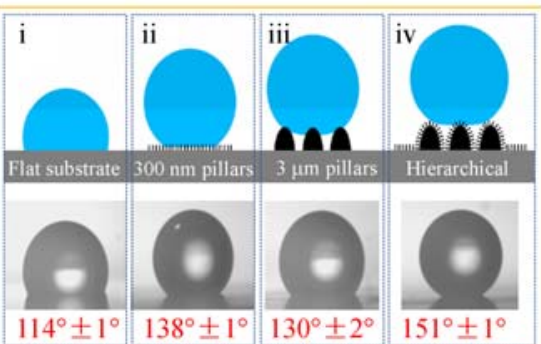

(b)

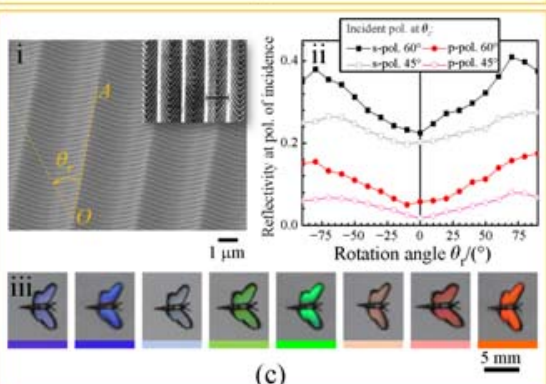

Fig. 6 (a) SEM micrographs of surfaces fabricated by multiple exposure of angle varied 2BI; (b) superhydrophobicity of hierarchical 3D textures; (c) optical properties of hierarchical 3D structure surfaces [5]. pol: Polarization

\section{Laser interference fabrication of micro/ nanostructures for light manipulation and nanoscale grown nanocrystals}

\subsection{Structures for optical manipulation}

High transmittance or low reflection is required in high- power laser systems, solar cells, and organic light emitting diodes (OLED) [40-42]. An antireflective coating by destructive interference is the usual option. However, this method has several problems, such as shedding by mechanical scratching, deformation from thermal expansion, and low-temperature shrinkage. These problems are critical, especially when the antireflective coatings are used 
for OLED, which are required to be flexible and stretchable in the future [43]. Inspired by the antireflective structure of moth eyes, structural antireflection through manufactured subwavelength structures was proposed by tuning the refractive index from step to gradual (shown in (iii) of Fig. 7(a)) [44-46]. Subwavelength periodic nanopillars were fabricated at bottom-emitting OLED windows by soft imprinting of periodic structures, which were fabricated by four-beam interference (shown in (i) and (ii) of Fig. 7(a)) [47-49]. The reversal structures had the same morphology as the original structures, with a period of approximately $440 \mathrm{~nm}$ and a height of approximately $500 \mathrm{~nm}$, which were sufficient to increase the transmittance from less than $91 \%$ to around $95 \%$ over a spectral range from 400 to $800 \mathrm{~nm}$, as shown in (iii) of Fig. 7(b). The enhancement was particularly evident when OLED operated in dirty environments due to the self-cleaning effect, which resulted in no dust particles remaining on OLED windows. This process significantly increased the transmittance from $50 \%$ (flat surface) to $95 \%$ (antireflective surface). This simple, cost-effective, and reproducible method provides large-area fabrication of antireflective and self-cleaning structures and may be applied in various applications for illumination and display [50-54].

Furthermore, light manipulation has attracted significant attention in recent years [55-59]. Laser interference technology is one of the promising techniques for the fabrication of 1D, 2D, isotropic, and anisotropic micro/ nanostructures for high-efficiency OLED [14,56,57,60]. Structures were usually built on the bottom and continued to the top, in which light was extracted. Light was efficiently coupled by plasmonic polaritons, especially when combined with metal structures [57]. In addition, structures with different periods were conducted for waveband manipulation in white light OLED $[61,62]$.

\subsection{Nano-limited effect for nanocrystal growth}

An application that combines superhydrophobicity and antireflection by using of hybrid micro-nanostructures is for high-resolution surface enhanced Raman spectrum (SERS) detection [4]. In addition, laser interference is widely used for template fabrication due to its nanofabrication capacities $[20,34,63]$. The minimum structure period is approximately half of the laser wavelength on the basis of the equation for laser interference period, which provides a way to realize the patterns of nanofeatures. As shown in Fig. 8, silver nanocrystals were grown through an electrochemical reaction with the aid of nanocells separated by SU8 photoresist. A nano-limited effect was observed, in which the nanocrystal was grown uniformly in each cell with the increase in etching time.
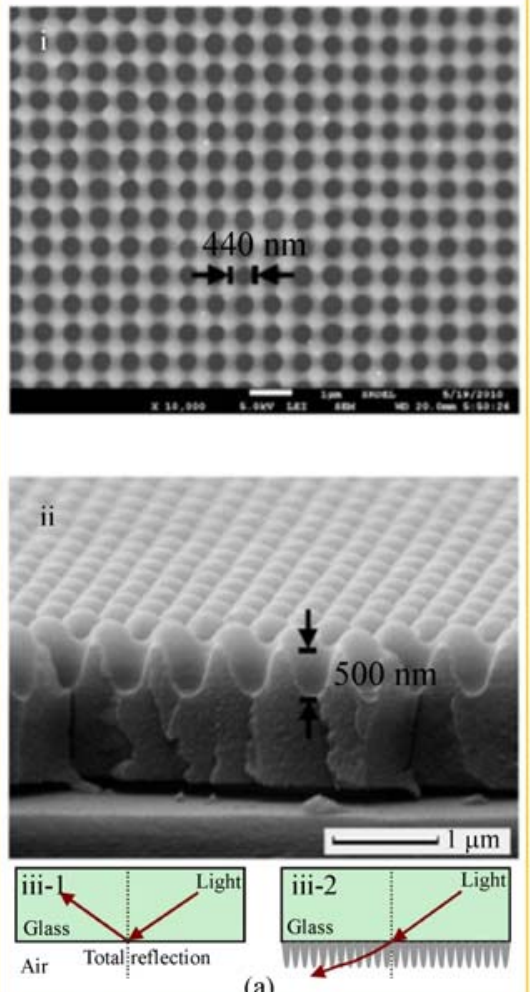

(a)

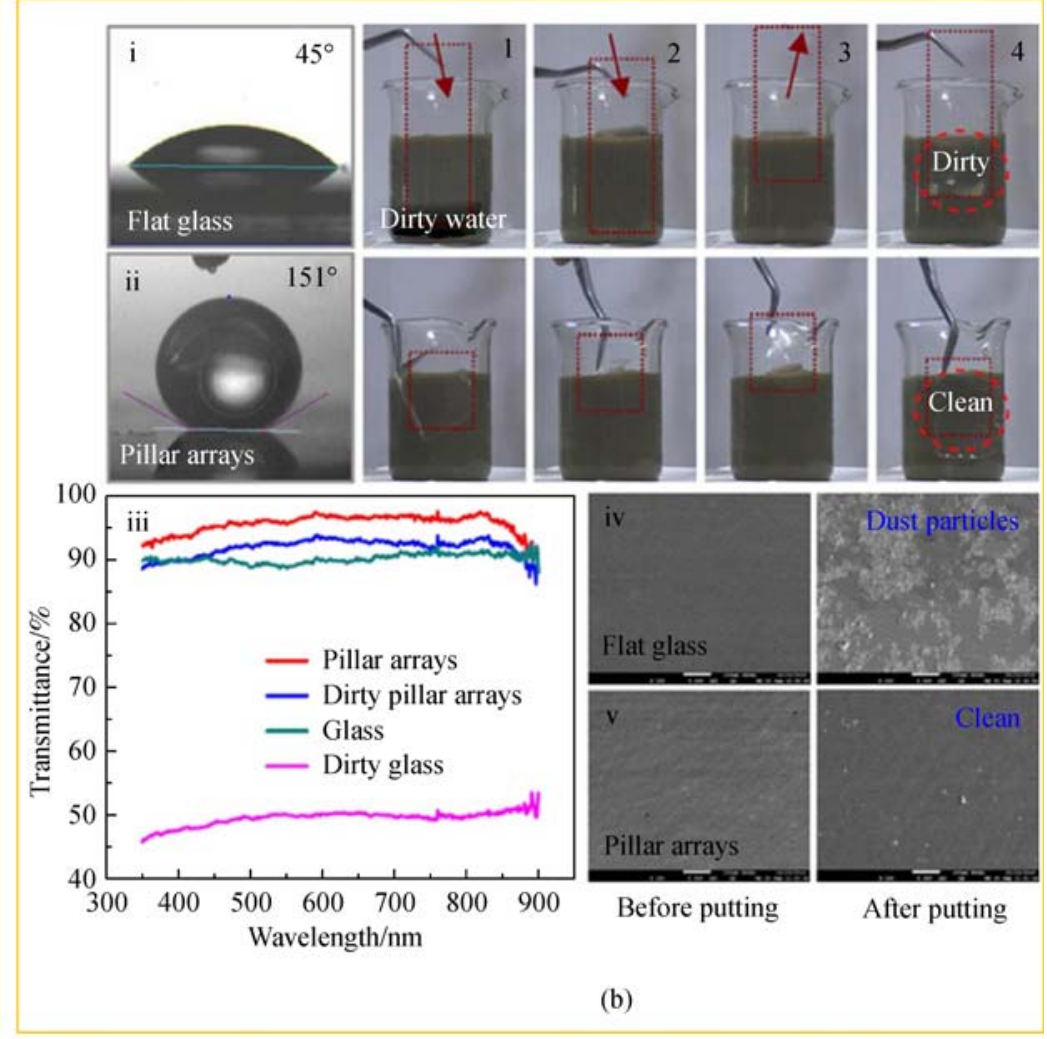

Fig. 7 Antireflective and self-cleaning for white organic light-emitting devices by flexible antireflective films. (a) Structure morphology (i) and (ii) and antireflection mechanism (iii); (b) self-cleaning superhydrophobic capability of nanopillar arrays [47] 


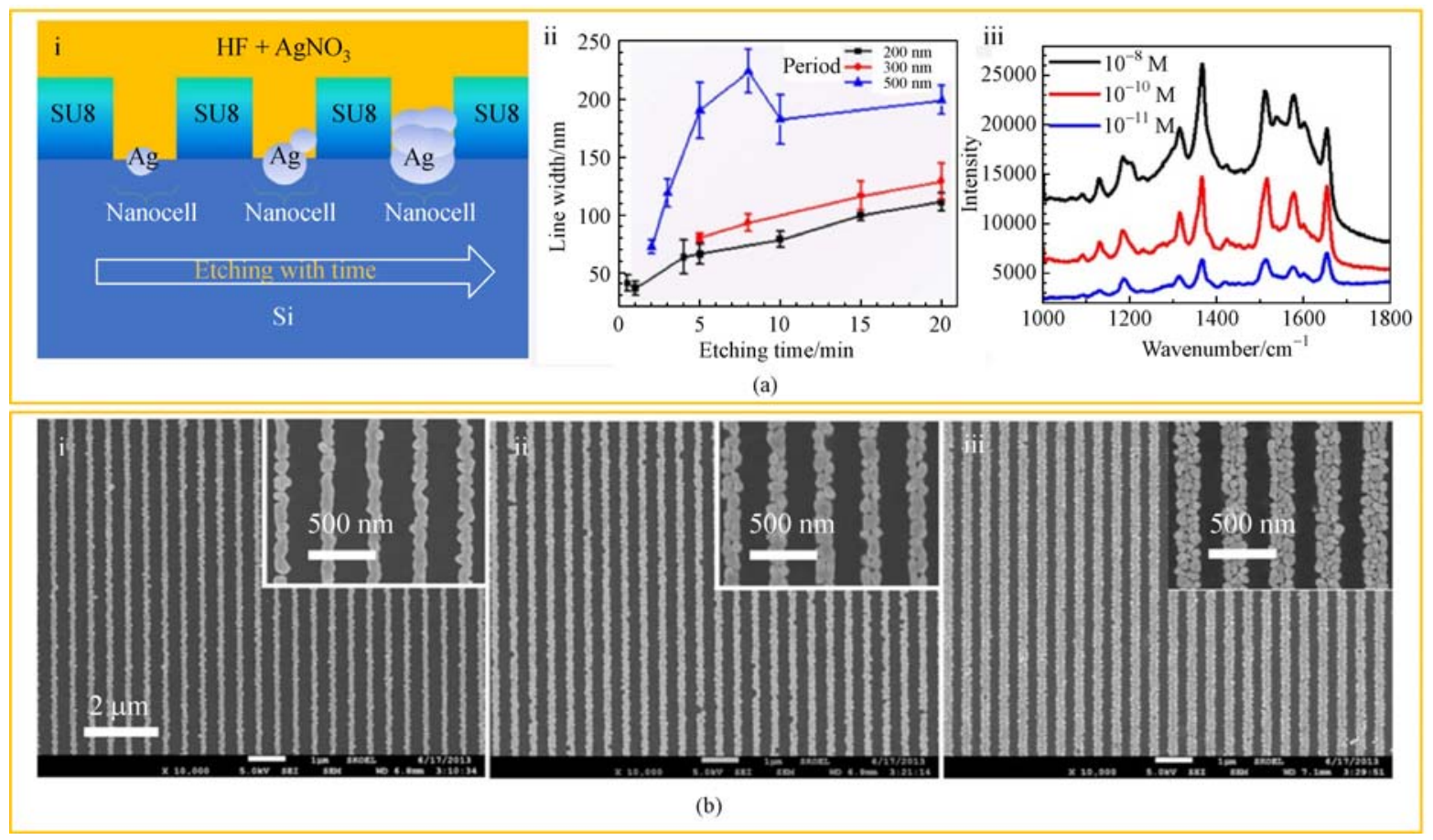

Fig. 8 (a) Schematic of nanocells limited controllable nanocrystal grown on Si for SERS; (b) the periodic silver nanocrystals grown on $\mathrm{Si}[64]$

Small nanogaps between periodic gratings makes the crystal growth slower and more uniform than those without limitation in experiments. A controllable and uniform shape and size could be realized, which was beneficial for surface enhanced Raman scattering detection and surface plasmon polariton excitation [64].

Generally, templates with a controllable period, line width, and dot size are widely used in laser interference technology due to their easily adjusted angles, light intensity, exposure time, and sample rotation $[6,65,66]$. Laser interference is a simple and widely used method for the fabrication of large-area and non-defect structures, which paves the way for the wide application of these periodic structures [66-69].

\section{Conclusions}

Laser interference is an efficient technology for the fabrication of periodic micro/nanostructures. Multiple beam interference and multiple exposure of two-beam interference are widely used. The former is dominant due to its flexibility in various structure fabrications. Tuning the laser fluence, angles, the exposure number between each beam, micropearl arrays, and anisotropic regular "S" and "chain-like" microstructures obtains a series of complex elliptical pillars and regular controllable microstructures by capillary force assembly of pillar arrays with different heights. Multiple two-beam interference pos- sesses simplicity and diversity, thus obtaining 1D, 2D, and hybrid 3D structures. The two structures fabricated by multiple beam interference and multiple exposure of twobeam interference have functional applications for antireflection, self-cleaning, and superhydrophobicity. Considering the complex experiment setup of multiple beam interference and its succenturiate in theory, multiple exposure of two-beam interference shows a broad potential for structure fabrication.

Laser inference technology has aimed to obtain many fine structures for nanoscience and surface science due to the period limitation of light interference. However, actually speaking, most of the structures by laser interference are between nano-region $(<100 \mathrm{~nm})$ to micro-region and even micro-region. In the future, immersion technology by interference in a high refractive index solution with deep ultraviolet light source are possible solutions to realize structure in nano-region. In addition, uniform morphology, especially for a large-area textured surface, has remained a challenge and is a research topic in the combination of nonlinear laser-matter interactions with ultrashort pulses.

Acknowledgements H. B. Sun thanks the National Key Research and Development Program of China and the National Natural Science Foundation of China (Grant Nos. 2017YFB1104300, 61590930, 20150203008GX, and 61605055).

Compliance with ethics guidelines All authors have no conflict of interest or financial conflicts to disclose. 
Open Access This article is distributed under the terms of the Creative Commons Attribution 4.0 International License (http://creativecommons.org/ licenses/by/4.0/), which permits unrestricted use, distribution, and reproduction in any medium, provided the appropriate credit is given to the original author(s) and the source, and a link is provided to the Creative Commons license, which indicates if changes are made.

\section{References}

1. Clapham P B, Hutley M C. Reduction of lens reflexion by the "moth eye" principle. Nature, 1973, 244(5414): 281-282

2. Li X M, Reinhoudt D, Crego-Calama M. What do we need for a superhydrophobic surface? A review on the recent progress in the preparation of superhydrophobic surfaces. Chemical Society Reviews, 2007, 36(8): 1350-1368

3. Roach P, Shirtcliffe N J, Newton M I. Progress in superhydrophobic surface development. Soft Matter, 2008, 4(2): 224-240

4. De Angelis F, Gentile F, Mecarini F, et al. Breaking the diffusion limit with super-hydrophobic delivery of molecules to plasmonic nanofocusing SERS structures. Nature Photonics, 2011, 5(11): 682687

5. Abid M I, Wang L, Chen Q D, et al. Angle-multiplexed optical printing of biomimetic hierarchical 3D textures. Laser \& Photonics Reviews, 2017, 11(2): 1600187

6. Xia D, Ku Z, Lee S C, et al. Nanostructures and functional materials fabricated by interferometric lithography. Advanced Materials, 2011, 23(2): 147-179

7. Kim K, Zhu W, Qu X, et al. 3D optical printing of piezoelectric nanoparticle-Polymer composite materials. ACS Nano, 2014, 8 (10): 9799-9806

8. Li Y, Zhang J, Zhu S, et al. Biomimetic surfaces for highperformance optics. Advanced Materials, 2009, 21(46): 47314734

9. Chen F. Micro- and submicrometric waveguiding structures in optical crystals produced by ion beams for photonic applications. Laser \& Photonics Reviews, 2012, 6(5): 622-640

10. Guo R, Yuan D, Das S. Large-area microlens arrays fabricated on flexible polycarbonate sheets via single-step laser interference ablation. Journal of Micromechanics and Microengineering, 2011, 21(1): 015010

11. Kim $\mathrm{K} \mathrm{S}$, Jeong $\mathrm{H}$, Jeong $\mathrm{M} \mathrm{S}$, et al. Polymer-templated hydrothermal growth of vertically aligned single-crystal $\mathrm{ZnO}$ nanorods and morphological transformations using structural polarity. Advanced Functional Materials, 2010, 20(18): 3055-3063

12. Wang L, Lu Z H, Lin X F, et al. Rapid fabrication of large-area periodic structures by multiple exposure of two-beam interference. Journal of Lightwave Technology, 2013, 31(2): 276-281

13. Lin C, Yan S, You F. Fabrication and characterization of shortperiod double-layer cross-grating with holographic lithography. Optics Communications, 2017, 383: 17-25

14. Liu Y F, Feng J, Yin D, et al. Viewing-angle independence of white emission from microcavity top-emitting organic light-emitting devices with periodically and gradually changed cavity length. Organic Electronics, 2013, 14(6): 1597-1601

15. Wu D, Chen Q D, Xia H, et al. A facile approach for artificial biomimetic surfaces with both superhydrophobicity and iridescence.
Soft Matter, 2010, 6(2): 263-267

16. Cho $\mathrm{C} \mathrm{Y}$, Moon J H. Hierarchically porous $\mathrm{TiO}_{2}$ electrodes fabricated by dual templating methods for dye-sensitized solar cells. Advanced Materials, 2011, 23(26): 2971-2975

17. Chen Y, Yuan D, Yang M, et al. High efficiency GaN LEDs with submicron-scale 2D periodic structures directly fabricated by laser interference ablation. Optics \& Laser Technology, 2017, 90: 211215

18. Wu D, Wu S Z, Chen Q D, et al. Curvature-driven reversible in situ switching between pinned and roll-down superhydrophobic states for water droplet transportation. Advanced Materials, 2011, 23(4): $545-549$

19. Wu D, Wu S, Chen Q D, et al. Facile creation of hierarchical PDMS microstructures with extreme underwater superoleophobicity for anti-oil application in microfluidic channels. Lab on a Chip, 2011, 11(22): 3873-3879

20. Cornago I, Hernández A L, Casquel R, et al. Bulk sensing performance comparison between silicon dioxide and resonant high aspect ratio nanopillars arrays fabricated by means of interference lithography. Optical Materials Express, 2016, 6(7): 2264-2272

21. Oh Y, Lim J W, Kim J G, et al. Plasmonic periodic nanodot arrays via laser interference lithography for organic photovoltaic cells with $>10 \%$ efficiency. ACS Nano, 2016, 10(11): 10143-10151

22. Raub A K, Li D, Frauenglass A, et al. Fabrication of $22 \mathrm{~nm}$ halfpitch silicon lines by single-exposure self-aligned spatial-frequency doubling. Journal of Vacuum Science \& Technology. B, Microelectronics and Nanometer Structures: Processing, Measurement, and Phenomena: An Official Journal of the American Vacuum Society, 2007, 25(6): 2224-2227

23. Kondo $\mathrm{T}$, Matsuo $\mathrm{S}$, Juodkazis $\mathrm{S}$, et al. Femtosecond laser interference technique with diffractive beam splitter for fabrication of three-dimensional photonic crystals. Applied Physics Letters, 2001, 79(6): 725-727

24. Lei M, Yao B L, Rupp R A. Structuring by multi-beam interference using symmetric pyramids. Optics Express, 2006, 14(12): 58035811

25. Wu D, Chen Q D, Xu B B, et al. Self-organization of polymer nanoneedles into large-area ordered flowerlike arrays. Applied Physics Letters, 2009, 95(9): 091902

26. Shoji S, Sun H B, Kawata S. Photofabrication of wood-pile threedimensional photonic crystals using four-beam laser interference. Applied Physics Letters, 2003, 83(4): 608-610

27. Kawai K, Sakamoto M, Noda K, et al. Tunable dichroic polarization beam splitter created by one-step holographic photoalignment using four-beam polarization interferometry. Journal of Applied Physics, 2017, 121(1): 013102

28. Shoji S, Sun H B, Kawata S. Photofabrication of wood-pile threedimensional photonic crystals using four-beam laser interference. Applied Physics Letters, 2003, 83(4): 608-610

29. Wang L, Cao X W, Li Q K, et al. Periodic structures fabricated by nanosecond laser four-beam interference ablation. Chinese Science Bulletin, 2016, 61(6): 616-621

30. Lee S K, Park S G, Moon J H, et al. Holographic fabrication of photonic nanostructures for optofluidic integration. Lab on a Chip, 2008, 8(3): 388-391

31. Dobrowolski J A, Guo Y N, Tiwald T, et al. Toward perfect 
antireflection coatings. 3. Experimental results obtained with the use of Reststrahlen materials. Applied Optics, 2006, 45(7): 1555-1562

32. Doshi P, Jellison G E, Rohatgi A. Characterization and optimization of absorbing plasma-enhanced chemical vapor deposited antireflection coatings for silicon photovoltaics. Applied Optics, 1997, 36 (30): 7826-7837

33. Ji L, Hsu H Y, Li X, et al. Localized dielectric breakdown and antireflection coating in metal-oxide-semiconductor photoelectrodes. Nature Materials, 2017, 16(1): 127-131

34. Yin D, Feng J, Ma R, et al. Efficient and mechanically robust stretchable organic light-emitting devices by a laser-programmable buckling process. Nature Communications, 2016, 7: 11573

35. Sahoo P K, Dev Choudhury B, Joseph J, et al. ZnO nanowireenabled light funneling effect for antireflection and light convergence applications. Optics Letters, 2017, 42(1): 45-48

36. Wang L, Xu B B, Chen Q D, et al. Maskless laser tailoring of conical pillar arrays for antireflective biomimetic surfaces. Optics Letters, 2011, 36(17): 3305-3307

37. Cai J, Qi L. Recent advances in antireflective surfaces based on nanostructure arrays. Materials Horizons, 2015, 2(1): 37-53

38. Wu D, Zhao Y B, Wu S Z, et al. Simultaneous efficiency enhancement and self-cleaning effect of white organic light-emitting devices by flexible antireflective films. Optics Letters, 2011, 36(14): 2635-2637

39. Wu D, Wu S Z, Chen Q D, et al. Curvature-driven reversible in situ switching between pinned and roll-down superhydrophobic states for water droplet transportation. Advanced Materials, 2011, 23(4): 545-549

40. Wu D, Chen Q D, Xu B B, et al. Self-organization of polymer nanoneedles into large-area ordered flowerlike arrays. Applied Physics Letters, 2009, 95(9): 091902

41. Kim J B, Lee J H, Moon C K, et al. Highly enhanced light extraction from surface plasmonic loss minimized organic light-emitting diodes. Advanced Materials, 2013, 25(26): 3571-3577

42. Zhu J, Hsu C M, Yu Z, et al. Nanodome solar cells with efficient light management and self-cleaning. Nano Letters, 2010, 10(6): 1979-1984

43. Rao J, Winfield R, Keeney L. Moth-eye-structured light-emitting diodes. Optics Communications, 2010, 283(11): 2446-2450

44. Qu Y, Coburn C, Fan D, et al. Elimination of plasmon losses and enhanced light extraction of top-emitting organic light-emitting devices using a reflective subelectrode grid. ACS Photonics, 2017, 4(2): 363-368

45. Forberich K, Dennler G, Scharber M C, et al. Performance improvement of organic solar cells with moth eye anti-reflection coating. Thin Solid Films, 2008, 516(20): 7167-7170

46. $\mathrm{Wu} \mathrm{S} \mathrm{Z,} \mathrm{Wu} \mathrm{D,} \mathrm{Yao} \mathrm{J,} \mathrm{et} \mathrm{al.} \mathrm{One-step} \mathrm{preparation} \mathrm{of} \mathrm{regular}$ micropearl arrays for two-direction controllable anisotropic wetting. Langmuir, 2010, 26(14): 12012-12016

47. Wu D, Wu S Z, Zhao S, et al. Rapid, controllable fabrication of regular complex microarchitectures by capillary assembly of micropillars and their application in selectively trapping/releasing microparticles. Small, 2013, 9(5): 760-767

48. Xu J, Wang Z, Zhang Z, et al. Fabrication of moth-eye structures on silicon by direct six-beam laser interference lithography. Journal of Applied Physics, 2014, 115(20): 203101
49. Burrow G M, Gaylord T K. Multi-beam interference advances and applications: Nano-electronics, photonic crystals, metamaterials, subwavelength structures, optical trapping, and biomedical structures. Micromachines, 2011, 2(4): 221-257

50. Lasagni A, Yuan D, Das S. Rapid fabrication of pentaerythritol triacrylate periodic structures on large areas by laser interference patterning with nanosecond pulses. Journal of Applied Physics, 2009, 105(2): 023101

51. Mao W, Liang G, Zou H, et al. Design and fabrication of twodimensional holographic photonic quasi crystals with high-order symmetries. JOSA B, 2006, 23(10): 2046-2050

52. Jiménez-Ceniceros A, Trejo-Durán M, Alvarado-Méndez E, et al. Extinction zones and scalability in N-beam interference lattices. Optics Communications, 2010, 283(3): 362-367

53. Abid M I, Wang L, Zhang X, et al. Silver nano islands enhanced Raman scattering on large area grating substrates fabricated by two beam laser interference. Chemical Research in Chinese Universities, 2013, 29(5): 1006-1010

54. Stavenga D G, Leertouwer H L, Osorio D C, et al. High refractive index of melanin in shiny occipital feathers of a bird of paradise. Light, Science \& Applications, 2015, 4(1): e243

55. Shi X B, Qian M, Zhou D Y, et al. Origin of light manipulation in nano-honeycomb structured organic light-emitting diodes. Journal of Materials Chemistry. C, Materials for Optical and Electronic Devices, 2015, 3(8): 1666-1671

56. Bi Y G, Feng J, Li Y F, et al. Broadband light extraction from white organic light-emitting devices by employing corrugated metallic electrodes with dual periodicity. Advanced Materials, 2013, 25(48): 6969-6974

57. Feng J, Liu Y F, Bi Y G, et al. Light manipulation in organic lightemitting devices by integrating micro/nano patterns. Laser \& Photonics Reviews, 2017, 11(2): 1600145

58. Ou Q D, Zhou L, Li Y Q, et al. Simultaneously enhancing color spatial uniformity and operational stability with deterministic quasiperiodic nanocone arrays for tandem organic light-emitting diodes. Advanced Optical Materials, 2015, 3(1): 87-94

59. Qiao W, Huang W, Liu Y, et al. Toward scalable flexible nanomanufacturing for photonic structures and devices. Advanced Materials, 2016, 28(47): 10353-10380

60. Choy W C H, Chan W K, Yuan Y. Recent advances in transition metal complexes and light-management engineering in organic optoelectronic devices. Advanced Materials, 2014, 26(31): 53685399

61. Bi Y G, Feng J, Chen Y, et al. Dual-periodic-corrugation-induced broadband light absorption enhancement in organic solar cells. Organic Electronics, 2015, 27: 167-172

62. Bi Y, Ji J, Chen Y, et al. Dual-periodic-microstructure-induced color tunable white organic light-emitting devices. Frontiers of Optoelectronics, 2016, 9(2): 283-289

63. Matioli E, Brinkley S, Kelchner K M, et al. High-brightness polarized light-emitting diodes. Light, Science \& Applications, 2012, 1(8): e22

64. Xu W, Okamoto T, Li A, et al. Preparation of large-area controllable patterned silver nanocrystals for high sensitive and stable surfaceenhanced Raman spectroscopy. Chemical Research in Chinese Universities, 2016, 32(3): 428-432 
65. Zhang J, Wang Z, Di X, et al. Effects of azimuthal angles on laser interference lithography. Applied Optics, 2014, 53(27): 62946301

66. Kravchenko A, Shevchenko A, Ovchinnikov V, et al. Optical interference lithography using azobenzene-functionalized polymers for micro- and nanopatterning of silicon. Advanced Materials, 2011, 23(36): 4174-4177

67. Bagheri S, Giessen H, Neubrech F. Large-area antenna-assisted
SEIRA substrates by laser interference lithography. Advanced Optical Materials, 2014, 2(11): 1050-1056

68. Moein T, Ji D, Zeng X, et al. Holographic photopolymer linear variable filter with enhanced blue reflection. ACS Applied Materials \& Interface, 2014, 6(5): 3081-3087

69. Gu F, Xie F, Lin X, et al. Single whispering-gallery mode lasing in polymer bottle microresonators via spatial pump engineering. Light, Science \& Applications, 2017, 6(10): e17061 